\title{
Mitochondrial dynamics regulates Drosophila intestinal stem cell differentiation
}

\author{
Hansong Deng $\mathbb{1}^{1,2}$, Shigeo Takashima ${ }^{3,4}$, Manash Paul ${ }^{3,5}$, Ming Guo ${ }^{2}$ and Volker Hartenstein ${ }^{3}$
}

\begin{abstract}
Differentiation of stem/progenitor cells is associated with a substantial increase in mitochondrial mass and complexity. Mitochondrial dynamics, including the processes of fusion and fission, plays an important role for somatic cell reprogramming and pluripotency maintenance in induced pluripotent cells (iPSCs). However, the role of mitochondrial dynamics during stem/progenitor cell differentiation in vivo remains elusive. Here we found differentiation of Drosophila intestinal stem cell is accompanied with continuous mitochondrial fusion. Mitochondrial fusion defective(opa1RNAi) ISCS contain less mitochondrial membrane potential, reduced ATP, and increased ROS level. Surprisingly, suppressing fusion also resulted in the failure of progenitor cells to differentiate. Cells did not switch on the expression of differentiation markers, and instead continued to show characteristics of progenitor cells. Meanwhile, proliferation or apoptosis was unaffected. The differentiation defect could be rescued by concomitant inhibition of Drp1, a mitochondrial fission molecule. Moreover, ROS scavenger also partially rescues opa1RNAiassociated differentiation defects via down-regulating JNK activity. We propose that mitochondrial fusion plays a pivotal role in controlling the developmental switch of stem cell fate.
\end{abstract}

\section{Introduction}

Stem differentiation is accompanied by pronounced changes in mitochondria. In embryonic stem cells (ESCs) or induced pluripotent stem cells (iPSCs), mitochondria are few in number, small and globular in shape, have fewer cristae, and are distributed as perinuclear clusters. During differentiation, mitochondria increase dramatically in mass and form an extensive tubular network ${ }^{1-5}$, while somatic cell reprogramming is accompanied by depletion of mitochondria through mitophagy (mitochondrial autophagy) ${ }^{6}$.

Correspondence: Hansong Deng (hdeng@tongji.edu.cn) or

Ming Guo (mingfly@ucla.edu) or Volker Hartenstein (volkerh@mcdb.ucla.edu)

${ }^{1}$ Shanghai East Hospital, School of Life Sciences and Technology, Tongji

University, Shanghai 20092, China

2Department of Neurology, Department of Molecular and Medical

Pharmacology, California Nanosystems Institute at UCLA David Geffen School of Medicine, University of California, Los Angeles, CA 90095, USA

Full list of author information is available at the end of the article.

These authors contributed equally Hansong Deng, Shigeo Takashima

Edited by I. Lavrik
For instance, cardio-myocyte differentiation in the embryonic heart is tightly linked to mitochondrial maturation ${ }^{7}$. Mouse hearts at embryonic day (E) 9.5 contain relatively few and immature mitochondria, characterized by rare and disorganized cristae. In E13.5 hearts the mitochondrial mass increases substantially, accompanied by maturation of the organelle as indicated by abundant laminar cristae ${ }^{7,8}$. Accordingly, one of the major obstacles for somatic reprogramming induced cardiomyocytes is to obtain functional mitochondria ${ }^{8}$. How these mitochondrial changes are regulated remains unexplored in vivo.

Mitochondrial morphology is regulated by continuous fusion and fission events. These dynamics processes are controlled by a group of GTPase proteins conserved from yeast to human. Mitofusins and opa1 are involved in outer and inner membrane fusion, respectively. Dynaminrelated protein 1 (Drp1) is responsible for mitochondrial fission. Previous results from our lab and others showed

\section{(c) 2018 The Author(s).}

(c) (i) Open Access This article is licensed under a Creative Commons Attribution 4.0 International License, which permits use, sharing, adaptation, distribution and reproduction cc) in any medium or format, as long as you give appropriate credit to the original author(s) and the source, provide a link to the Creative Commons license, and indicate if changes were made. The images or other third party material in this article are included in the article's Creative Commons license, unless indicated otherwise in a credit line to the material. If material is not included in the article's Creative Commons license and your intended use is not permitted by statutory regulation or exceeds the permitted use, you will need to obtain permission directly from the copyright holder. To view a copy of this license, visit http://creativecommons.org/licenses/by/4.0/. 
that Drosophila homologs of fusion (MARF/mfn, opa1) and fission (drp1) are functionally conserved and are involved in pathogenesis of neuro-degeneration diseases $^{9-12}$. Recent findings indicated that mitochondrial fission is required for stem cell pluripotency maintenance in iPSCs and $\mathrm{ESCs}^{13}$. Aside from affecting energy metabolism, mitochondrial fusion and fission has many other effects on developing and mature cells, in particular in regard to mitochondrial DNA (mtDNA) distribution, ROS production, calcium overload, and apoptosis ${ }^{14-16}$. However, the role of mitochondrial dynamics on stem cell differentiation in developing context is largely unknown.

The Drosophila adult gut and its stem cell populations derive from gut progenitors of the larval stage. In the midgut, these cells form small clusters (adult midgut progenitors or AMPs) scattered over the outer surface of the midgut epithelium ${ }^{17,18}$; in the hindgut, adult progenitors form a narrow domain, the hindgut proliferation zone (HPZ) also known as imaginal ring, located near the anterior hindgut boundary ${ }^{19,20}$ (Fig. 1a). During early metamorphosis, these progenitor populations expand over the larval gut which undergoes programmed cell death. Subsequently, most progenitors differentiate into adult enterocytes, whereas a small subpopulation of progenitors is held back and gives rise to adult progenitor/ stem cells (Fig. 1a). These cells are quiescent in nature but are highly inducible upon tissue damage ${ }^{19,21}$. We speculated that mitochondrial dynamics and the functional changes they engender controls the switch from gut progenitor to differentiated enterocytes.

\section{Results}

Mitochondria undergo a dramatic increase in volume and complexity during differentiation of Drosophila intestinal cells

Mitochondria of hindgut cells were labeled by the UASmito-GFP transgene driven by byn-GAL4, a hindgutspecific driver ${ }^{22,23}$ (Fig. 1e-i and Fig. S1A). For the midgut, the esg-Gal4 driver was used ${ }^{17}$ (Fig. S2A and S2B). In the HPZ domain, the GFP-labeled mitochondria are few, small in volume, and perinuclear in location (Fig. 1e, f and $\left.\mathrm{h}-\mathrm{h}^{\prime}\right)$. AMPs of the larva also possess small, granular mitochondria (Fig. S2A). Mitochondria of the differentiated hindgut and midgut enterocytes have strongly increased in volume and number (Fig. 1e, g, i- $\mathrm{i}^{\prime}$ and Fig. S2B). Transmission electron microscopy (TEM) was used to resolve mitochondrial morphology and structure at a higher-level resolution. The adult progenitor/stem cells in the HPZ domain located adjacent to the malpighian tubes and the mitochondria in these cells are small and round in shape and cristae is rarely observed (Fig. 1c). Mitochondria of midgut progenitors have similar dimensions (Fig. S2C). Differentiated adult hindgut enterocytes have enlarged in size and form a thick cuticle layer on the apical membrane facing the gut lumen; apical membranes are deeply invaginated, and mitochondria are located in between membrane invaginations (Fig. 1c and c '). Mitochondria are densely packed, elongated apicobasally, and often highly branched (Fig. 1c and $\mathrm{c}^{\prime}$ ). The size of mitochondria in these areas varies from 200 to 500 $\mathrm{nm}$ in diameter, and often several microns in length. Differentiated midgut enterocytes do not form cuticle, but a dense array of microvilli was formed at their apical pole. Mitochondria take up most of the cytoplasm of these cells; as in the hindgut, individual mitochondria are much larger, elongated, and often branched (Fig.S2D).

\section{Inhibiting mitochondrial fusion through opa1 or $\mathrm{mfn}$ knockdown causes hindgut stem/progenitor cells failed to differentiate}

To manipulate mitochondrial dynamics, we took advantage of RNA interference (RNAi) and of overexpression of the responsible genes, using the UAS-GAL4 system and the temperature-sensitive GAL80 repressor $\left(\mathrm{GAL}^{\mathrm{ts}}{ }^{\mathrm{ts}}\right)$. Byn-Gal4-directed expression of an opa1RNAi construct was able to block mitochondrial fusion in the hindgut. Mitochondria in opa1-RNAi-expressing hindgut enterocytes are ellipsoid in shape (Fig. $1 \mathrm{~d}-\mathrm{d}^{\prime}$ and Fig. S1C), a similar phenomenon was observed in marf RNAi hindguts (Fig. S1B). Aside from the expected defect in mitochondrial fusion, opa1-RNAi severely affected hindgut development. Byn-Gal4 > opa-1 RNAi animals die within 2 days after eclosion (Fig. 2a), although the eclosion rate is comparable with the sibling controls (data not shown). Similar result was obtained when opa1RNAi was knocked down from the L1 stage using the GAL80 ${ }^{\text {ts }}$ system, ruling out the possibility of embryogenesis mispatterning. The hindgut is significantly shorter and slightly "fatter" compared with control flies (Fig. 2b, c).

Stat92E-GFP (stat-GFP in short), a reporter for JAK-STAT pathway activity ${ }^{24}$ whose expression is restricted to the HPZ in wild-type hindguts (Fig. 2d), remains strongly expressed throughout the entire hindgut of opa-1 RNAi animals (Fig. 2e). Enterocytes is surrounded by basal circular muscles in the hindgut. The apical membrane inviginations and cuticles of enterocytes can be densely stained by Toluidine blue (Fig. 2i). However, the prospective enterocytes in opa1 RNAi are highly dilated and no apical membrane invaginations or cuticle was formed inside (Fig. 1d, $d^{\prime}$ and 2j). The acute lethality of opa1-RNA flies after eclosion and cellular structural abnormality in enterocytes suggested the lack of functionally differentiated cells. Indeed, opa-1(RNAi) hindguts lack signs of differentiation. By in situ hybridization, we found that FSH (CG7665), which is highly expressed in differentiated hindgut enterocytes of wild-type flies, is reduced or absent in opa-1 (RNAi) flies (Fig. 2n, o). Similar phenomena were found in byn-GAL4>marf RNAi flies (Fig. $2 \mathrm{~h}, \mathrm{~m}$ and $\mathrm{r}$ ). To test 


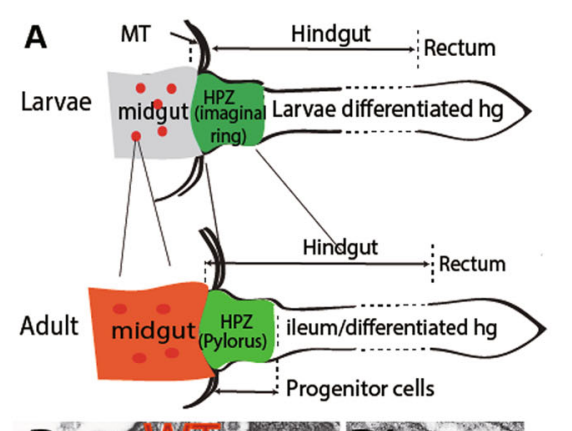

\section{BynGal4, UASmitoGFP}
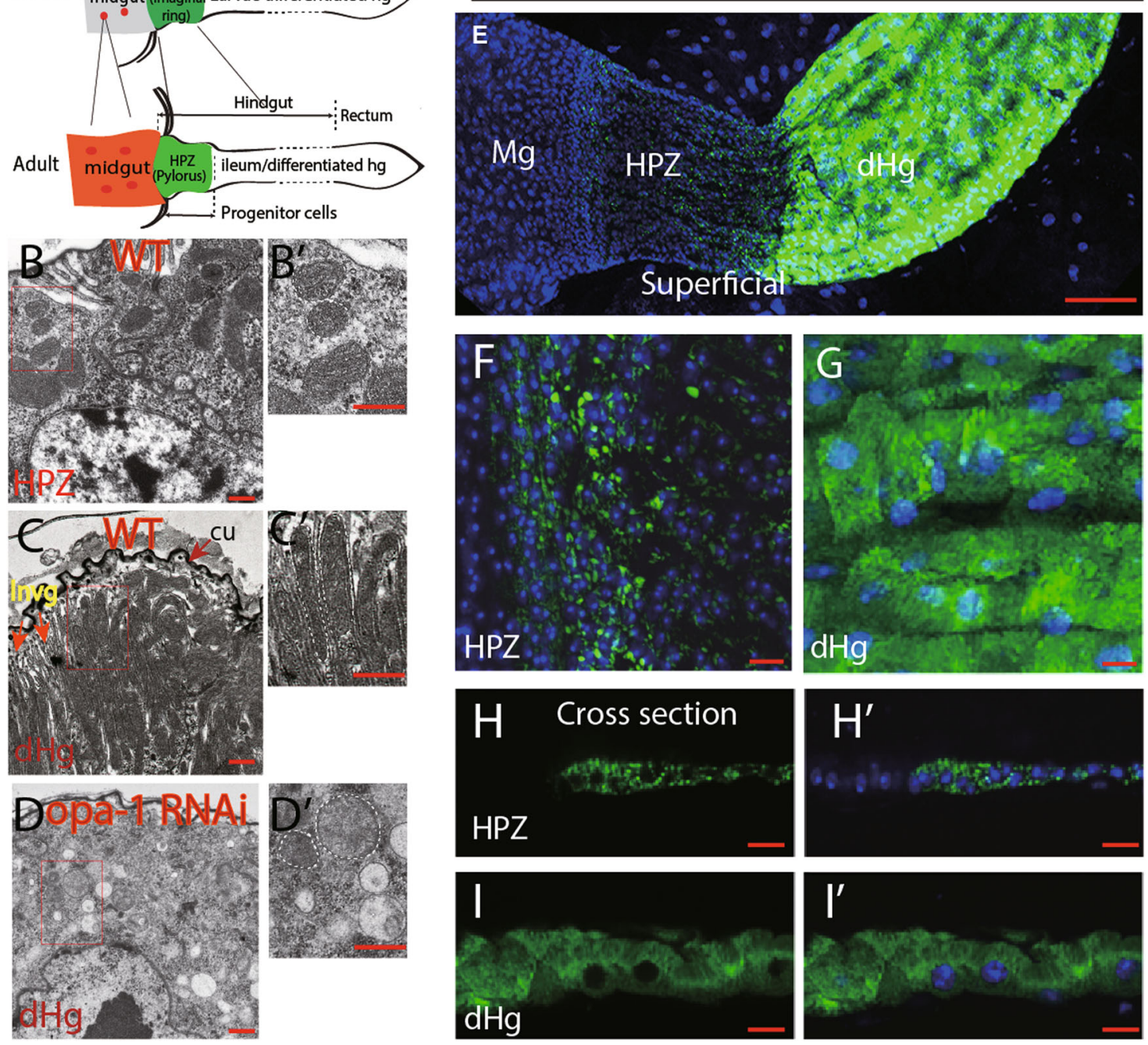

Fig. 1 Extensive mitochondrial fusion during hindgut differentiation and opa-1 RNAi inhibit the fusion process. a Schematic representation of gut development in Drosophila. Progenitors in the larvae stage give rise to the adult gut including its stem cell/progenitor populations. Midgut and hindgut are connected through Malpighian tubes (MT). During early metamorphosis, the progenitor populations expand over the larval gut, which undergoes programmed cell death. For instance, in the midgut, adult midgut progenitors (AMPs, denoted in red dots) in the larvae stage generate adult gut and stem cells (red dots). Similarly, hindgut proliferative zone (HPZ) cells (also called as hindgut imaginal ring, denoted in green) in the larvae stage generates the whole adult hindgut (green), including a subset of progenitors in the anterior hindgut (adult HPZ/Pylorus) and the differentiated hindgut (also called as ileum). Please refer to the main text for details. Mitochondria morphology of hindgut cells in different regions was observed by TEM from $\mathbf{b}$ to $\mathbf{d}$ and by confocal microscopy from e to i. b Mitochondria in adult HPZ cells. Note that the HPZ cells identity was based on the physical location and their unique morphology. c Mitochondria in adult differentiated cells. The matured enterocytes form a thick layer of cuticle structure (" $\mathrm{cu}$ " in brief) toward the lumen. Mitochondria aligned with membrane invigination ("invg" in brief). $\mathbf{d}$ Mitochondria in BynGal4>opa1 RNAi adult differentiated cells. For $\mathbf{b}-\mathbf{d}$, higher magnification of rectangle area shown on the right in $\mathbf{b}^{\prime}-\mathbf{d}^{\prime}$. Mitochondrial borders are marked with dashed lines. Cu cuticle, invg invigination, dHg differentiated hindgut, MT Malpighian tubes. e-i Mitochondria morphology visualized by byn-GAL4 > UAS-mito-GFP under confocal microscopy. $\mathbf{f}$ (HPZ domain) and $\mathbf{g}$ (differentiation hindgut, $\mathrm{dHg}$ ) are higher magnification of $\mathbf{e}$. $\mathbf{h}-\mathbf{h}^{\prime}$ and $\mathbf{i}-\mathbf{i}^{\prime}$ are cross-sectional view of HPZ and dHg cells, respectively. From e to i, TOTO3 labels nuclei in blue. Scale bars: $200 \mu \mathrm{m}$ in $\mathbf{b}-\mathbf{d}$, $100 \mu \mathrm{m}$ for e, $20 \mu \mathrm{m}$ for $\mathbf{f}-\mathbf{i}$

whether an increase in mitochondrial fusion also causes hindgut dysfunction, we knocked down Drp1, an essential component of the mitochondrial fission machinery ${ }^{25,26}$. Expression of drp1-RNAi in the hindgut elicited a definitive change in the mito-GFP signal, suggesting irregular and enlarged mitochondria (Fig. S1D). However, the viability of drp1-RNAi animals is comparable to wild type (Fig. 2a). Cellular structure such as apical membrane invagination 


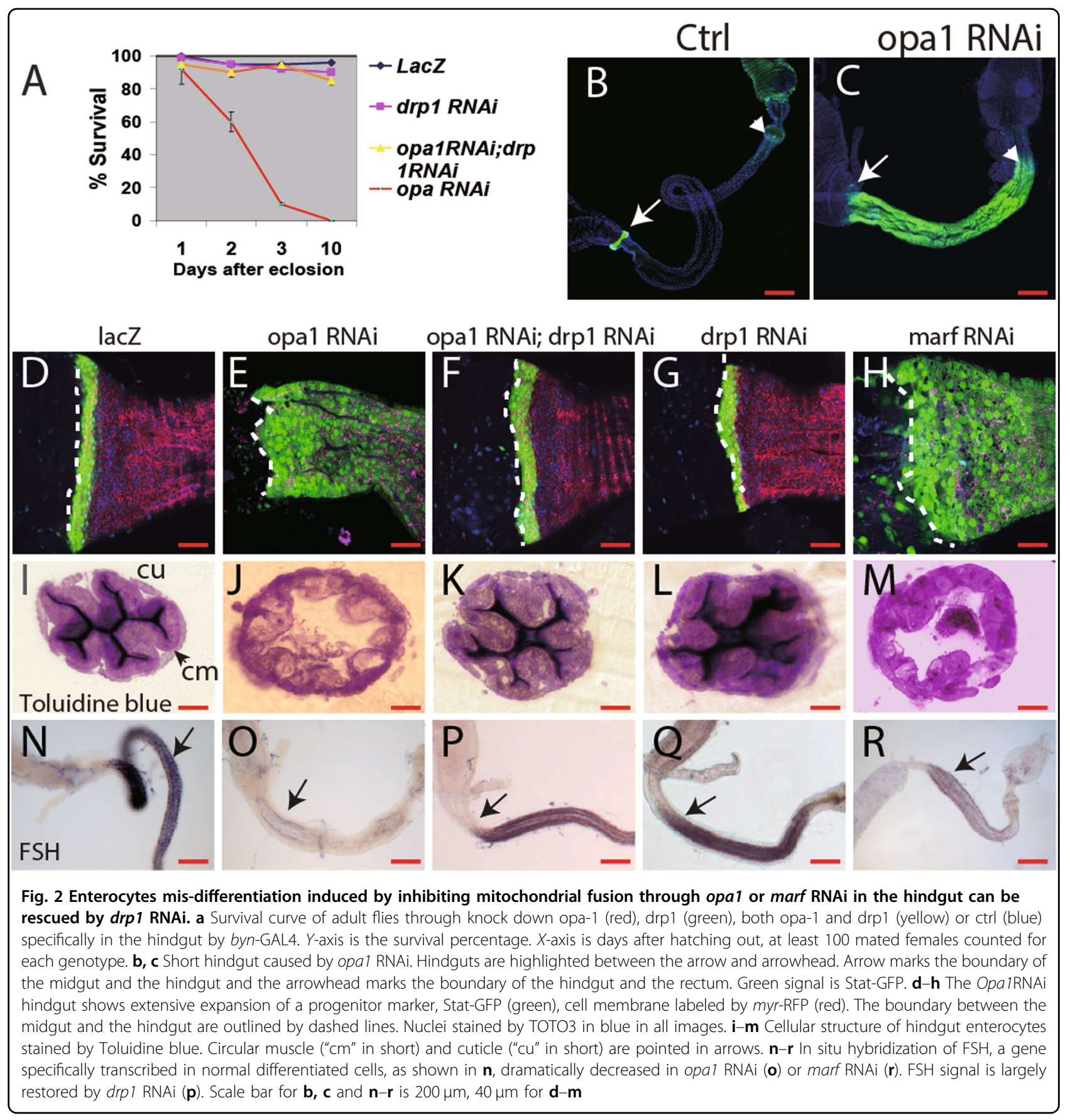

and cuticle as well as Stat-GFP expression are not significantly altered (Fig. 2g, l). Over-expression of the fusion gene Marf also produced no obvious defect on hindgut marker expression or cellular structure, although the mitochondria are more elongated than in control flies (data not shown). These results suggested that loss of fission or over-activation of fusion is dispensable for hindgut function. Next, we wanted to test if defects caused by opa-1 RNAi and marf RNAi can be rescued by reduced fission (drp1 RNAi) or over-fusion (marf OE). Indeed, the acute lethality of opa1-RNAi flies can be fully rescued by drp1 knockdown (Fig. 2a and data not shown). Importantly, the hindguts of the double knockdowns were properly elongated and expressed nearly normal pattern of Stat-GFP (Fig. 2f, k). Furthermore, enterocyte differentiation failure in the opa1RNAi hindgut can be restored by drp1RNAi: the hindgut of the double knockdowns expressed nearly normal level of FSH (Fig. 2p, q). Similar results were obtained by simultaneously knocking down Marf and drp1 (data not shown). 


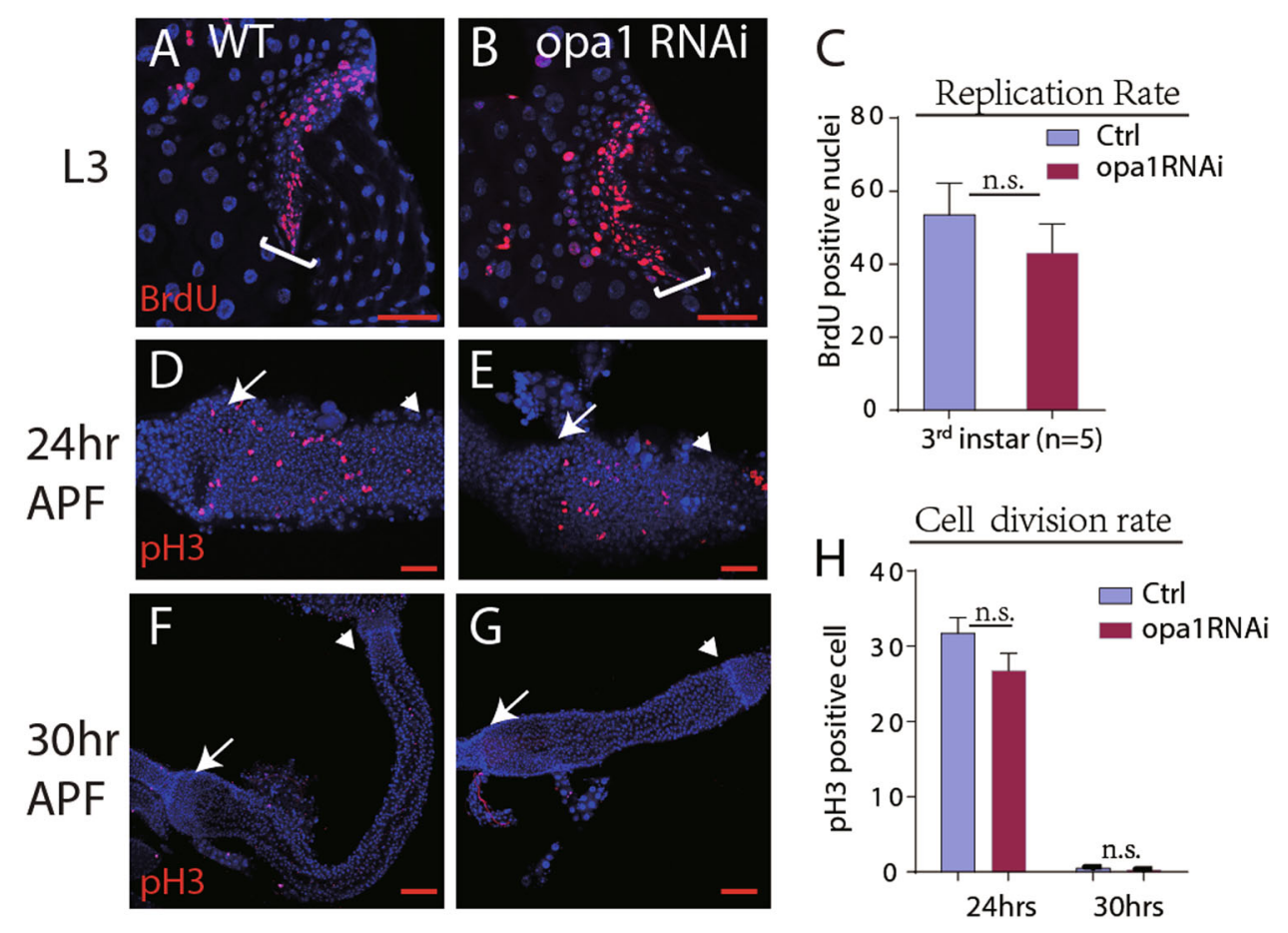

Fig. 3 Stem/progenitor cell proliferation is largely unaffected by opa1RNAi. a-c Replication rate was measured by BrdU feeding in stagematched third larvae. Representative images are shown as $\mathbf{a}$ (ctrl) and $\mathbf{b}$ (opal RNAi). The HPZ zone is outlined with a white bracket. Quantification shown in $\mathbf{c}$. No statistical significance was found, $n=5$. $\mathbf{d}$-h Proliferation rate was indicated by pH3-positive nuclei in larvae hindgut in $24 \mathrm{~h}$ APF (d, e) and $30 \mathrm{~h}$ APF $(\mathbf{f}, \mathbf{g})$. $\mathbf{d}$ and $\mathbf{f}$ are the Controls; e and $\mathbf{g}$ are opa-1 RNAi larvae. Quantification shown in $\mathbf{h}$. No statistical significance was found, $n=6$. Error bars represent standard deviation (STDEV) in $\mathbf{c}$ and $\mathbf{h}$. Arrows pointed to the boundary of the midgut and the hindgut, and TOTO3 stained nuclei in blue. Scale bar for $100 \mu \mathrm{m}$

\section{Stem/progenitor cell proliferation is largely unaffected by opa1RNAi}

As described above, the progenitor cell maker, StatGFP, expanded in the whole hindgut in opa-1(RNAi) and marf (RNAi) flies. We hypothesized that loss of mitochondrial fusion may trigger stem cell over-proliferation and form a stem cell like tumor. As a result, stem cells could fail to differentiate properly. To test this hypothesis, we analyzed proliferation by applying BrdU, which is specifically incorporated in DNA of S phase cells. Adult opa-1(RNAi) flies were fed BrdU-containing food for $24 \mathrm{~h}$. After staining, we did not find a significant difference in the number of BrdU-containing cells between control and opa-1(RNAi) flies (data not shown). Proliferation was also tested during the larval stage. Mid-third instar larvae were raised on BrdU-containing food for $24 \mathrm{~h}$. We found an average of 55 BrdU-positive cells in control larvae, and 50 in opa1-RNAi larvae; the difference is not significant $(n=$ 6; Fig. 3a-c). Proliferation of the adult hindgut progenitors normally ceases around $24 \mathrm{~h}$ after puparium formation (apf) ${ }^{27}$. To rule out that the phase of proliferation is extended in opa1-RNAi, we monitored pupae $24 \mathrm{~h}$ apf and $30 \mathrm{~h}$ apf, using an antibody against phosphorylated histone 3 (pH3). At 24h, opa1-RNAi hindguts showed the same number of mitotic cells as controls (Fig. 3d, e and h). Likewise, at $30 \mathrm{~h}$ apf, opa1-RNAi guts were devoid of mitotic cells, similar to controls (Fig. $3 \mathrm{f}-\mathrm{h}$ ). Wg acts as an essential signal to stimulate proliferation of the HPZ and at the same time inhibits differentiation ${ }^{20}$. Overexpression of $\mathrm{Wg}$ in the HPZ results in a hindgut phenotype that, in regard to lack of enterocyte differentiation, resembles the opa1-RNAi phenotype. To investigate whether knock down of opal affects wg expression, we used a Wg-lacZ reporter construct in the background of byn-Gal4-driven opa1-RNAi. No significant change in the pattern of Wg-lacZ expression is evident (Fig. S3A and S3B). Furthermore, CG31607, which normally transcribed only in HPZ cells, has no or slight expansion in opa-1 (RNAi) flies (Fig. S3C and S3D). Taken together, these results suggest that the block of mitochondrial fusion by opa1-RNAi did not affect proliferation in the developing intestine.

The hindgut defects in opa-1(RNAi) flies are not caused by apoptosis of differentiated enterocytes

Mitochondrial dynamics is closely related to apopto$\operatorname{sis}^{14}$, and we wondered whether the opa1 and marf knockdown phenotype is due to excessive cell death of 
enterocytes. Our findings speak against this hypothesis. No obvious increase of apoptotic cells was found in the opa-1 RNAi hindgut by TUNEL staining (terminal deoxynucleotidyl transferase dUTP nick end labeling) (Fig. S4A and S4B). As a control, we found excessive TUNEL-positive cells when an rpr; hid over-expression construct was induced in the hindgut (Fig. S4C). Furthermore, over-expression of p35 and Diap, two caspase inhibitors $^{28}$, failed to rescue the opa-1(RNAi) defects in terms of lethality or ectopic stat-GFP in the hindgut (Figures S4D-F and data not shown). These data suggest that the absence of differentiated cells in opa-1(RNAi) flies is due to a failure of differentiation, rather than apoptosis of differentiated cells.

\section{Mitochondrial defects induced by inhibiting mitochondrial fusion in hindgut}

The functional output of mitochondria can be monitored by the mitochondrial membrane potential, which reflects the proton gradient generated by oxidative phosphorylation across the inner membrane. Tetramethylrhodamine ethyl ester (TMRE) is a commonly used dye to monitor mitochondria membrane potential in live cells $^{29}$. We found that the increase in mitochondrial number and volume during enterocyte differentiation is accompanied by an increase in mitochondrial membrane potential. Hindgut enterocyte progenitors exhibit low TMRE signal, compared with the differentiated adult cells (Fig. 4a/a'/a" and Fig. S5A-C). Knocking-down opa1 caused a reduced functional output of mitochondria. The membrane potential dropped to a level that, quantitatively, corresponded to that of enterocyte progenitors (compare panels Fig. $4 a^{\prime} / a^{\prime \prime}$ and $b^{\prime} / b^{\prime \prime}$ ). Significant decrease of membrane potential was also found in opa1 RNAi clones (Fig. $4 \mathrm{c} / \mathrm{c}^{\prime} / \mathrm{c}^{\prime \prime}$ ). Mitochondrial ATP production is coupled with membrane potential ${ }^{30}$. As expected, opa1 RNAi hindguts have significantly lower ATP level than controls. Although drp1 knocking down also decrease ATP production slightly, drp1 RNAi significantly restores ATP level production of opa1 RNAi hindguts (Fig. 4d).

\section{Excessive ROS induced JNK activity contributes to mis- differentiation in opa1RNAi hindgut}

We and others previously showed that ROS function as a signal molecule controls stem cell proliferation in Drosophila ISCs and mammalian airway basal stem cells $(\text { ABSCs })^{31,32}$. Since most of ROS are produced by mitochondria and are closely regulated by mitochondrial fis$\operatorname{sion}^{14}$. We sought to test whether ROS is involved in hindgut defects of opa1RNAi flies.

Dihydroethidium (DHE) was used as a specific dye to measure the ROS level in freshly dissected guts. DHE fluorescence is relatively lower in the HPZ zone, while higher in differentiated cells in control hindguts (Figs. 5a and $\mathrm{a}^{\prime}$ ). However, in opa1-RNAi hindguts, all hindgut cells have a relatively higher ROS level (Note: 'black holes' likely due to non-apoptotic cell death in opa1RNAi hindguts, shown as asterisk in Fig. 5b and $\mathrm{b}^{\prime}$ ). It implies that elevated ROS production may cause differentiation defects in opa1RNAi hindguts. As expected, overexpressing Jafrac1, a thioredoxin peroxidase 1, led to a significantly reduction of ROS level and suppressed nonapoptotic cell death phenotype in opa1RNAi hindguts (Fig.5c and $\mathrm{c}^{\prime}$ ).

JNK mediated multiple downstream effects of ROS signaling ${ }^{31,33}$. Puc-LacZ was used as a reporter for JNK activity $^{33}$. We found that opa1RNAi hindguts have much higher Puc-LacZ staining than controls and can be fully suppressed by overexpressing a dominant-negative form of JNK, UASBsk ${ }^{\text {DN }}$ (Fig. 5d, f). Moreover, UASBsk ${ }^{\text {DN }}$ can partially suppress opa1RNAi-associated differentiation defects, such as StatGFP expansion and gut length (Fig. $5 g-i)$.

All these results indicated that the ROS-JNK pathway participated in opa1RNAi hindgut defects.

\section{Inhibit mitochondrial fission also inhibit midgut enterocyte differentiation}

Next, we examined the requirement of fusion for adult midgut differentiation. Larvae expressing opa1 RNAi under the esg-GAL4 driver from the early (L1) stage onward fail to reach the pupal stage and arrest at the third instar or pre-pupal stage (data not shown), probably because of the multiple-tissue expression of esg-GAL4 which may cause pleiotropic defects in development ${ }^{34}$. Differentiation defects was observed in the midgut progenitors of these animals as well. In wild type, midgut progenitors split into two cell types. The center of each progenitor cluster is occupied by small, rounded midgut progenitors which will form the adult midgut. These cells are surrounded by large, flattened peripheral cells which during early metamorphosis differentiate into a transient pupal midgut ${ }^{35,36}$ (Fig. S6A and B). Following opa1-RNAi expression in midgut progenitors, peripheral cells did not differentiate (Fig. S6C). However, drp1 knocking down fully suppressed the differentiation defects (Fig. S6D and E). To overcome the early lethality, we expressed opa 1 RNAi from the late third instar stage. Under these conditions, larvae can pupate and eclose. Examination of midguts of freshly eclosed adults revealed a phenotype that resembled in many aspects of the above described hindgut phenotype. A large proportion of midgut cells shows signs of immaturity, in terms of small size, continued expression of esg, and Stat-GFP, another ISC/EB marker ${ }^{35,37}$ (Fig. 6a-e). Moreover, the expression of Delta also showed massive expansion along with Esg in opa1 RNAi flies (Fig. S7A, B). On the other hand, Pdm-1, a 

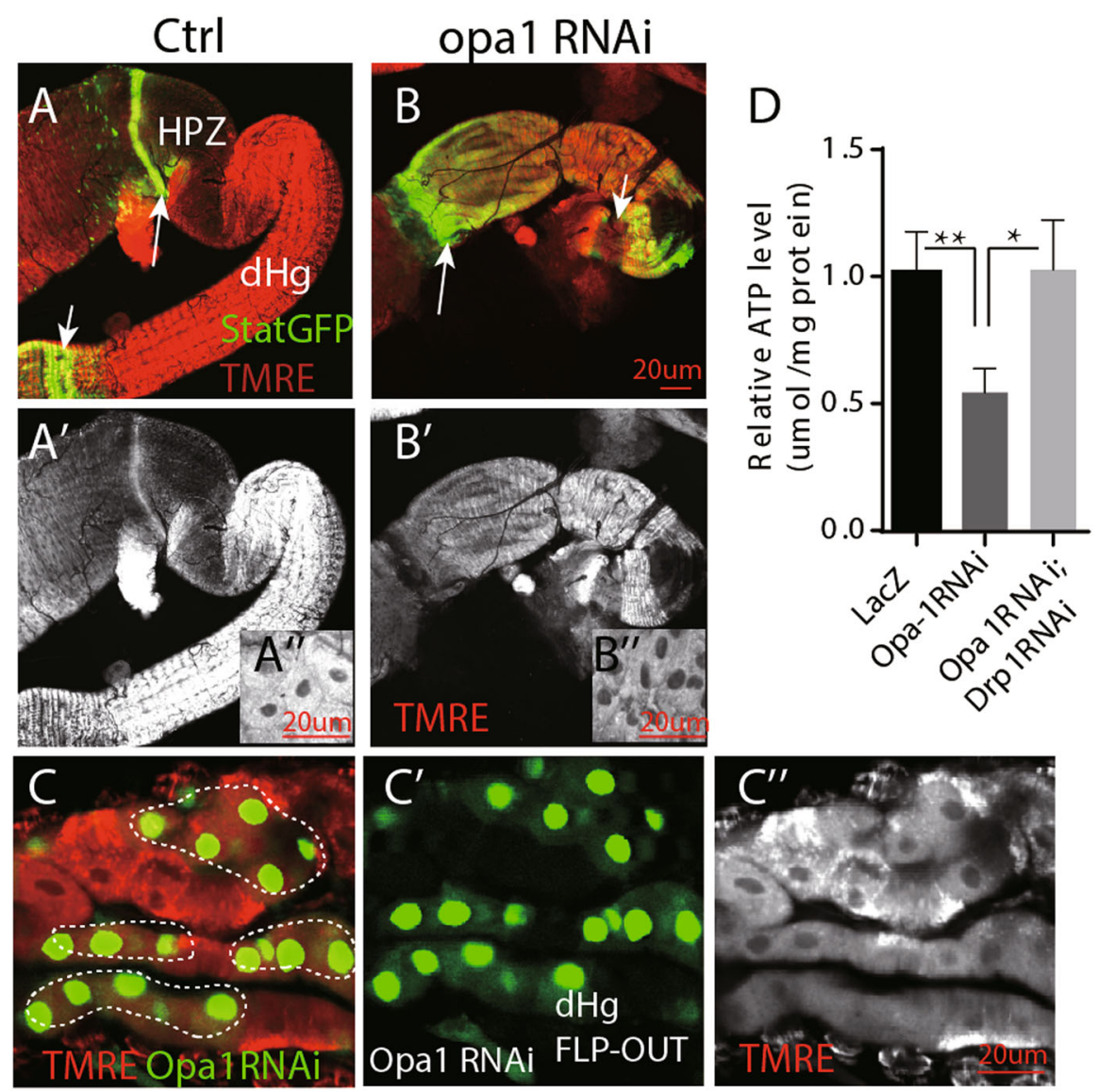

Fig. 4 Mitochondrial defects induced by inhibiting mitochondrial fusion through opa 1RNAi in hindgut. a, b TMRE staining in freshly dissected adult hindgut. Note wild-type enterocytes ("En" in short) have much higher fluorescent staining than cells in the HPZ domain. $\mathbf{a}^{\prime}$ and $\mathbf{b}^{\prime}$ are separate TMRE channels. $\mathbf{a}^{\prime \prime \prime}$ and $\mathbf{b}^{\prime \prime \prime}$ are higher magnified image of rectangle area in $\mathbf{a}^{\prime}$ and $\mathbf{b}^{\prime}$, respectively. The arrows point to the boundary of midgut and hindgut. The arrowheads denote the boundary of the hindgut and the rectum. c Opal RNAi clones in the adult hindgut have much lower membrane potential by TMRE staining. Clones are marked by dashed lines. $\mathbf{c}^{\prime}$ and $\mathbf{c}^{\prime \prime}$ are separate GFP and TMRE channel, respectively. Genotype: hsFLP; UASGFP; Tub < tub80 > GAL4/opa1 RNAi. d Quantification of a relative ATP level of isolated hindguts with different genotypes: Ctrl, opa1 RNAi, and opa1 RNAi; drp 1 RNAi. Error bars represent standard deviation (STDEV). Scale bar is $20 \mu \mathrm{m}$

differentiation marker for enterocyte ${ }^{38,39}$, was normally expressed in most of these cells (data not shown). We speculated that the relatively late onset of opa1 RNAi expression (see above) may in part be responsible for the weak differentiation phenotype, compared to the hindgut. We therefore examined the effect of opa1 knock-down FLP-out clones. First instar larvae, with the genotype of $h s \mathrm{FLP}$; tub > GAL80 > GAL4, UAS-GFP/UAS-opa1RNAi, were heat-shocked at $37^{\circ} \mathrm{C}$ for $1 \mathrm{~h}$ to induce opa1 RNAiexpressing clones and the phenotype was examined at the adult stage. Clones of cells knocked-down for opa1 had no or decreased level of the differentiation marker Pdm-1, compared with clones without opa-1 RNAi (Fig. 6f- $\mathrm{f}^{\prime \prime \prime}$ ). Meanwhile, clones bearing multiple copies of opa1RNAi induced in adult by another lineage tracing stock (esg ${ }^{\text {ts }}$ GFP; UAS-FLP, tub < CD2 > GAL4) also showed smaller and significantly weaker expression of Pdm1 (Fig. $\left.6 g-g^{\prime \prime \prime}\right)$. Matured enterocytes often migrated toward the apical gut lumen and form actin enriched microvilli, which was stained by phalloidin. In control GFP-positive clones, enterocytes are adjacent to the phalloidin-stained structure (Fig. 6h); however, the enterocytes retained in the basal side in opa1 RNAi clones (Fig. 6i). Finally, the cell size in the opa1RNAi clones is much smaller compared with the adjacent non-labeled differentiated cells (Fig. 6j), indicating the immature differentiation. Again, this defect can be rescued by drp1 knock-down (Fig. 6k, l).

\section{Discussion}

Our results here indicated that mitochondrial fusion, followed by increased functional output, forms part of the causal chain that switches the cells state from stem/progenitor cell towards differentiation. Thus, preventing fusion by knock-down of opa-1 or marf can block enterocyte differentiation in intestinal stem/progenitor cells. On the other hand, blocking fission by over-expression of marf or knock 

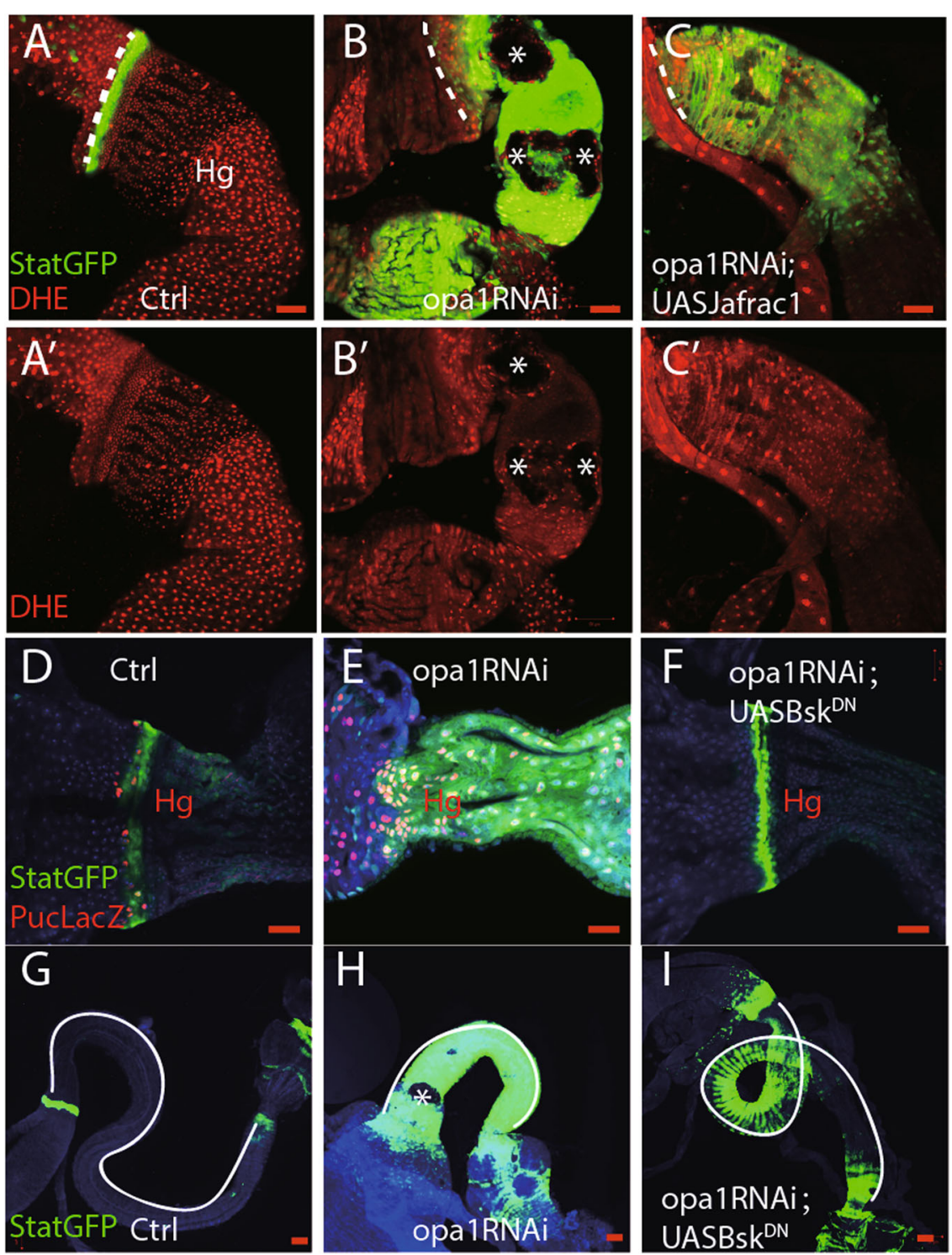

Fig. 5 ROS-JNK pathway contribute to differentiation defects in opa1RNAi hindguts. a-c DHE staining of freshly dissected hindgut. $\mathbf{a}^{\prime}-\mathbf{c}^{\prime}$ are DHE channel. Asterisk denotes potentially non-apoptotic cell death in opalRNAi hindguts. $\mathbf{d}-\mathbf{f}$ Anti-beta Gal staining against Puc-LacZ in different genotypes. g-i Differentiation index, such as Stat::GFP and gut length, was compared in different conditions. Gut length was delineated out by white lines. Scale bar is $20 \mu \mathrm{m}$

down drp1 did not cause obvious defects in differentiation. Moreover, blocking fission by knocking down of drp-1 rescued the defects of opa-1 RNAi. We then demonstrated that mitochondria in the opal RNAi hindgut have lower membrane potential, less ATP production, and higher ROS. We then showed that a high ROS level contribute to misdifferentiation in opa1RNAi hindgut by increasing JNK activity. These findings indicate that mitochondrial fusion is critical for enterocyte differentiation (Fig. 7).

ROS, in conjunction with the JNK pathway, affects stem cell activity, cell fate transition and cell survival ${ }^{40}$. Our previous results showed that in mouse and human ABSCs, low to moderate ROS levels is required for stem cell selfrenewal and proliferation ${ }^{31}$. Similarly, Drosophila midgut stem cells have relatively lower ROS level compared with differentiated enterocytes and increasing ROS level promotes proliferation rate ${ }^{32}$. Here, we showed that ROSmediated JNK pathway contributed to opa1RNAi hindgut mis-differentiation, indicating intrinsic difference of stem cells in response to ROS signaling. Besides ROS, mitochondrial fusion could also trigger retrograde signaling pathways such as calcium ${ }^{41}$, which we recently described to capable to alter stem cell activity in Drosophila midgut $\mathrm{ISCs}^{42}$ 


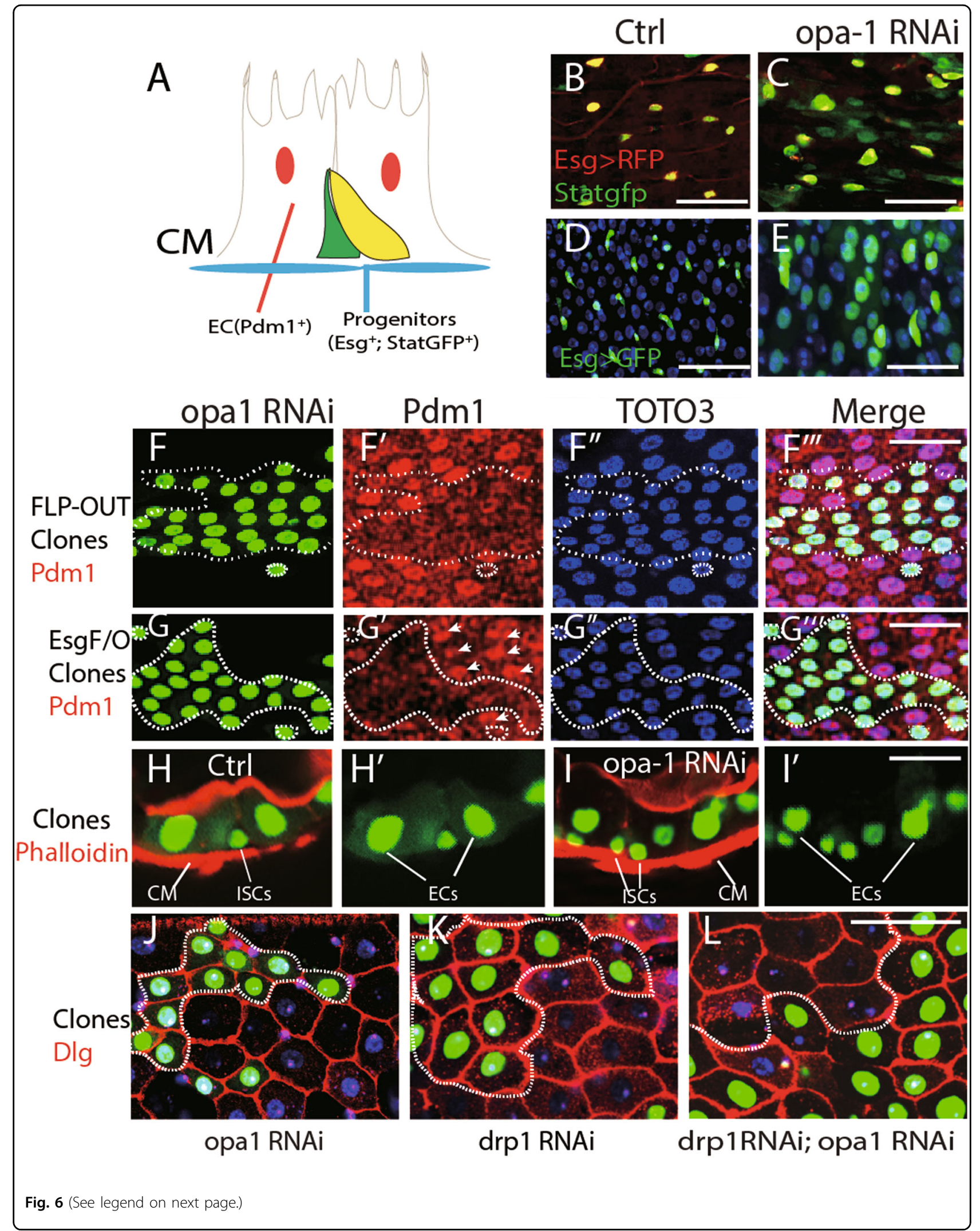


(see figure on previous page)

Fig. 6 Inhibiting mitochondrial fission also impairs midgut enterocyte differentiation. a Schematic view of cell types in the adult posterior midgut. Basal located progenitor cells (green) are Esg and Stat-GFP positive. Enlarged enterocytes can be labeled by differentiation marker Pdm1. CM is the brief for circular muscle. Both circular muscle and actin enriched microvilli in enterocytes can be stained by Phalloidin. b, c Ectopic Stat-GFPpositive cells (green) in the opa-1 RNAi midgut. The progenitor cells in the normal midgut are labeled with membrane-bound UAS-myr-RFP driven by Esg-GAL4. d, e Massive enterocyte-like cells are Esg positive (green) in the opa-1 RNAi midgut. In normal midgut, Esg-positive diploid cells are labeled by esg-GAL4; UASGFP (d). f Opa-1 RNAi Flp-out clones induced in the larvae stage have less Pdm-1 expression in the adult midgut. GFP-positive clones are outlined with dashed lines. $\mathbf{f}^{\prime} \mathbf{f}^{\prime}, \mathbf{f}^{\prime \prime}$, and $\mathbf{f}^{\prime \prime \prime}$ are GFP, Pdm1, TOTO3, and Merged channel, respectively. $\mathbf{g}$ Opa-1 RNAi clones induced in the adult stage have less Pdm-1 expression in the adult midgut. GFP-positive clones are marked with dashed lines. $\mathbf{g}, \mathbf{g}^{\prime}, \mathbf{g}^{\prime \prime}$, and $\mathbf{g}^{\prime \prime \prime}$ are GFP, Pdm1, TOTO3, and Merged channel, respectively. $\mathbf{h}$, $\mathbf{i}$ Mis-differentiation of enterocytes in opar RNAi clones. Circular muscle is "CM" in short. Actin enriched microvilli and circular muscles are stained with phalloidin in red. $\mathbf{h}^{\prime}$ and $\mathbf{i}^{\prime}$ are GFP channel of $\mathbf{h}$ and $\mathbf{i}$, respectively. $\mathbf{j}$-I Enterocytes in opal RNAi clones are smaller in size. GFP-positive clones are labeled with dashed lines. The cell boundary was stained with Dlg in red. TOTO3 labels nuclei in blue. Scale bar is $20 \mu \mathrm{m}$. Genotype for $\mathbf{f}$ and $\mathbf{i}$ : hsFLP; UASGFP; Tub < tub80 > GAL4/opa1 RNAi. Genotype for $\mathbf{g}$ : hsFLP; esg-GAL4, UASGFP, TubGAL80; ${ }^{\text {ts }}$ Tub < tub80 > GAL4/opa1 RNAi Genotype for h: hsFLP; UASGFP; Tub < tub80 > GAL4 Genotype for $\mathbf{k}$ : hsFLP; UASGFP; Tub < tub80 > GAL4/drp1 RNAi Genotype for I: hsFLP; UASGFP; Tub < tub80 > GAL4/drp1 RNAi, opa1 RNAi

\section{Mitochondrial fusion and biogenesis}

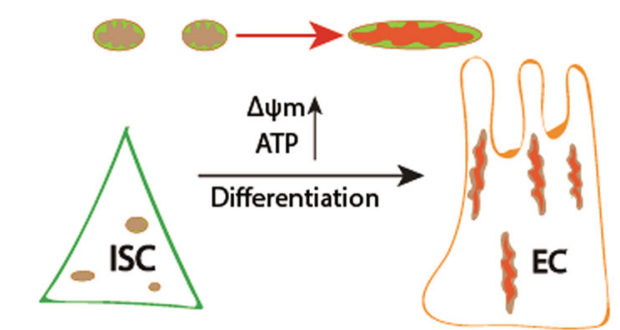

\section{Loss of fusion( opa1RNAi or mfn RNAi)}

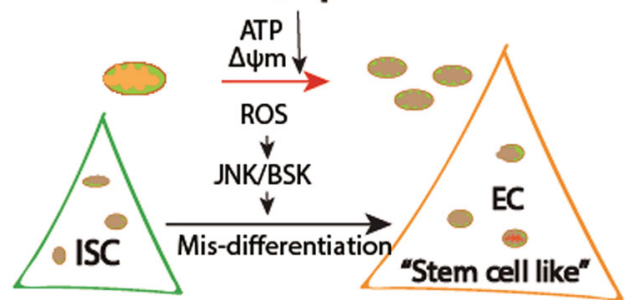

Fig. 7 Model on mitochondrial fission-mediated differentiation failure in Drosophila intestine stem cells. Proliferative cells, including Drosophila intestine stem cells in this scenario, bearing hypo-active, smaller and fewer mitochondria with having a relative lower membrane potential (marked in purple). Hence, self-renewal is largely independent on mitochondria function. During differentiation, mitochondria continuously undergo fusion and biogenesis to cope with the energy demand. Correspondingly, mitochondria became more active (marked in bright red). Meanwhile, mitochondria may send some retrograde signals, such as ROS, ATP, and/or some unknown molecules back to nucleus to program the differentiation process. On the other hand, loss of fusion by opa-1 RNAi or marf RNAi causes mis-differentiation of progenitor cells. The "prospective" differentiated cells are much smaller and still express progenitor marker (statGFP) but not differentiated marker (FSH). Loss of fusion can block production

In addition to these retrograde signaling processes, mitochondrial fusion might also cause energetic changes (ATP level) that affect differentiation. Although genetically and pharmacologically augment of cellular ATP level fail to rescue the mis-differentiation in opa1 RNAi flies (data not shown), we cannot exclude the possibility that optimal cellular level of ATP regulates stem cell differentiation.

Taken together, we described that the net effect of mitochondrial dynamics in Drosophila intestine stem cell lineage is a progressive fusion process and this process is essential for stem cell differentiation. Considering mitochondrial maturation is also crucial for iPSCs differentiation, our results suggested that boosting mitochondrial fusion could produce more functionally differentiated cells and can be targeted for regenerative medicine.

\section{Materials and methods}

\section{Fly stocks and genetics}

Flies used in this study were (donors in parentheses) byn-Gal4 (J. Lengyel), hh-Gal4 (K. Basler), esg-GAL4 (N. Perrimon) UAS-P35, UAS-Diap (B. Hay), Esg ${ }^{\text {ts }}$ GFP (B. Edgar), 10xStat92E-GFP (E. Bach), UASJafrac1(H. Jasper) and the following stocks were obtained from Bloomington stock center: tub-GAL80 ${ }^{\text {ts }}$, Ser-GAL4, UAS-mito-GFP, $H s F L P$, Aygal4, UASGFP or from the National Institute of Genetics, Japan: $\mathrm{Wg}$-lacZ/CyO. All flies were reared with normal fly food at room temperature or in incubators at $18^{\circ} \mathrm{C}, 25^{\circ} \mathrm{C}$, or $29^{\circ} \mathrm{C}$. FLP-OUT clones were induced at late second instar or early third instar larvae by heat shock $1 \mathrm{~h}$ at $37^{\circ} \mathrm{C}$ water bath. Clones are marked with GFP. For hindgut differentiation experiments, early second instar larvae were shift from $18^{\circ} \mathrm{C}-22^{\circ} \mathrm{C}$ to $29^{\circ} \mathrm{C}$. The hindguts were dissected 2 days after hatching out.

\section{Transgene construct}

UASsmDRP1: To silence DRP1, coding region in the DRP1 was targeted using a microRNA-based technology $^{43}$. PCR products of an microRNA precursor was cloned into pUAST. All cloned PCR products were confirmed by DNA sequencing ${ }^{43}$. 


\section{Survival assay}

Around 100 mated female flies for each genotype were counted, which were raised and kept in $29^{\circ} \mathrm{C}$ from first larvae on. Dead flies were scored each day in 2 weeks. Three independent experiments were performed.

\section{BrdU incorporation assay}

Labelling proliferative cells with BrdU was performed by feeding adult flies or larvae with BrdU. Adult flies of 1 week after eclosion or older were reared with normal fly food containing BrdU ( $1 \mathrm{mg} / \mathrm{ml}$, Sigma) for 3 or 7 days consecutively. Third instar larvae were fed with the same food containing BrdU for $2-4 \mathrm{~h}$. Hindguts of adults or larvae were dissected and fixed immediately after BrdU labelling or subsequently reared with normal fly food without BrdU for another 7-14 days before dissection, and then treated with $2 \mathrm{~N} \mathrm{HCl}$ for $30 \mathrm{~min}$ on ice, before being processed for antibody staining.

\section{Toluidine blue staining and TEM}

The hindgut was excised under a dissection microscope, fixed with $2.5 \%$ glutaraldehyde in $0.05 \mathrm{M}$ phosphate buffer (pH 7.4) for $1.5 \mathrm{~h}$ at $4{ }^{\circ} \mathrm{C}$, and washed three times with 0.05 $\mathrm{M}$ phosphate buffer at $4{ }^{\circ} \mathrm{C}$, post-fixed with $1.0 \% \mathrm{OsO}_{4}$ for $1 \mathrm{~h}$ at $4{ }^{\circ} \mathrm{C}$, washed with phosphate buffer, dehydrated in ethanol, and embedded in Spurr resin. Thick section was stained with Toluidine blue and ultra-thin sections of the embedded guts were double-stained with uranyl acetate and lead citrate and examined with a JEOL $100 \mathrm{C}$ transmission electron microscope (TEM).

\section{In situ hybridization}

In situ hybridization of FSH is followed as described ${ }^{44}$. Briefly, dissected hindguts were fixed with $4 \%$ formaldehyde and stored in $100 \%$ methanol at $-20^{\circ} \mathrm{C}$ until use. The hindguts were rehydrated with a descending methanol series and treated with PBS containing 0.1\% Tween 20 (PBS-tw). They were then reacted with $10 \mu \mathrm{g} /$ $\mathrm{mL}$ of proteinase $\mathrm{K}$ diluted with PBS-tw; the reaction was stopped with $2 \mathrm{mg} / \mathrm{mL}$ of glycine dissolved in PBS-tw. After being fixed with $4 \%$ formaldehyde again and washed with PBS-tw, hindguts were hybridized with a digoxygenin-labeled RNA probe prepared against FSH or CG31607 cDNA diluted with hybridization buffer at $60^{\circ} \mathrm{C}$ for overnight. After washing out non-hybridized probes with $50 \%$ formamide diluted with $10 \times$ SSCT, $2 \times$ SSCT, and $0.2 \times$ SSCT (performed at $60{ }^{\circ} \mathrm{C}$ ) and then briefly washed with PBS-tw, they were blocked with $0.2 \%$ Blocking reagent (Roche, Indianapolis, IN) diluted with PBS-tw and then treated with anti-Digoxygenin antibody labeled with alkaline-phosphatase (Roche) overnight. After washing with PBS-tw, the hybridized probe was detected by NBT/BCIP (tablet, Roche). The primer sequence of FSH is: CACGGGGCTGAAGGTCTACG GAT and TGCAGCCAAGCCCGTACTGCCAA.

CG31607 is now called CG43394 according to the FlyBase and its probe was synthesized from a plasmid obtained from DGRC and the plasmid \# is RE17733.

\section{Antibody staining}

Hindguts and midgut were fixed with $4 \%$ formaldehyde/PBT for $45 \mathrm{~min}$ at room temperature and then treated with primary antibody overnight at $4{ }^{\circ} \mathrm{C}$. After treatment with secondary antibody conjugated with fluorescent dye, hindguts and midguts were mounted with Vectashield (Sigma). A nuclear indicator (TOTO3) was added to the mounting medium if necessary (1:2000 dilutions). Antibodies used in this study were: mouse anti-Arm (1:10; DSHB, University of Iowa), mouse anti- $\beta$-gal (1:100; Promega), Mouse antiCycA, mouse anti-CycB (1:5; DSHB), mouse antiLaminC (1:50; DSHB), Rabbit Phosho-H3 (1:100; Molecular Probes), Mouse anti-Delta (1:50; DSHB), and rabbit anti-Pdm1 (gift from Xiaohang Yang, Institute of Molecular and Cell Biology, Singapore).

\section{Mitochondria membrane potential measurement}

For membrane potential, whole gut was dissected in Schneider solution at room temperature, then stained in Schneider solution containing $1 \mu \mathrm{M}$ TMRE (dissolved in $100 \%$ ethanol, from Molecular Probes) for $20 \mathrm{~min}$, washed in Schneider $2 \times 5 \mathrm{~min}$, and then, directly imaged under a confocal microscope. For ROS staining, DHE staining was followed as described ${ }^{32}$. In brief, fresh guts were dissected and incubated in Schneider medium containing $50 \mu \mathrm{M}$ DHE (dissolved in DMSO, from Molecular Probes) for 20 min, washed in Schneider $2 \times 5 \mathrm{~min}$, and then, directly imaged under a confocal microscope. All these experiments were carried out in dark.

\section{ATP measurement}

The hindgut ATP level was measured using a luciferasebased bioluminescence assay (ATP Bioluminescence Assay Kit HS II; Roche Applied Science). For each measurement, ten hindguts were freshly dissected out (with midgut removed) and immediately homogenized in $50 \mu \mathrm{l}$ lysis buffer. The lysate was boiled for $5 \mathrm{~min}$ and cleared by centrifugation at $20,000 \mathrm{~g}$ for $1 \mathrm{~min}$. Five microliters of cleared lysate was added to $90 \mu \mathrm{l}$ dilution buffer and $5 \mu \mathrm{l}$ luciferase, and the luminescence was immediately measured using a 96-well plate luminometer. Each reading was converted to the amount of ATP per hindgut based on the standard curve generated with ATP standards. The readings will then be normalized with the protein level measured by BCA Bradford assay. Three measurements were made for each genotype. 


\section{Acknowledgements}

We thank Dr. Haixia Huang in Dr. Bruce Hay's lab for generating UASsmDRP1 construct. We also thank Bruce Edgar, Utpal Banerjee, Erika Bach, Norbert Perrimon, Bruce Hay, Konrad Basler, Henri Jasper, and NIG (Japan), Bloomington (USA) Drosophila Stock Centers for fly stocks; Xiaohang Yang for anti-Pdm-1 antibody. This work was supported by National Key R\&D Projects (2018YFA0107100), Youth 1000 Talent Plan of China and Tongji University Basic Scientific Research-Interdisciplinary Fund to H.D., NIH grant R01 NS054814 To V.H., and funds of NIH AG033410 and NINDS EUREKA award, the Glenn Family Foundation, Natalie R. and Eugene S. Jones Fund in Aging and Neurodegenerative Disease Research, Louis B. Mayer Foundation and McKnight Neuroscience Foundation to M.G.

\section{Author details}

'Shanghai East Hospital, School of Life Sciences and Technology, Tongji University, Shanghai 20092, China. ${ }^{2}$ Department of Neurology, Department of Molecular and Medical Pharmacology, California Nanosystems Institute at UCLA David Geffen School of Medicine, University of California, Los Angeles, CA 90095, USA. ${ }^{3}$ Department of Molecular Cell and Developmental Biology, University of California Los Angeles, Los Angeles, CA 90095, USA. ${ }^{\text {P}}$ resent address: Life Science Research Center, Gifu University, Gifu 501-1194, Japan. ${ }^{5}$ Present address: Division of Pulmonary and Critical Care Medicine, David Geffen School of Medicine, University of California, Los Angeles, CA 90095, USA

\section{Conflict of interest}

The authors declare that they have no conflict of interest.

\section{Publisher's note}

Springer Nature remains neutral with regard to jurisdictional claims in published maps and institutional affiliations.

The online version of this article (https://doi.org/10.1038/s41420-018-0083-0) contains supplementary material, which is available to authorized users.

Received: 16 April 2018 Revised: 21 May 2018 Accepted: 22 June 2018 Published online: 23 July 2018

\section{References}

1. Schieke, S. M. et al. Mitochondrial metabolism modulates differentiation and teratoma formation capacity in mouse embryonic stem cells. J. Biol. Chem. 283, 28506-28512 (2008).

2. Varum, S. et al. Enhancement of human embryonic stem cell pluripotency through inhibition of the mitochondrial respiratory chain. Stem Cell Res. 3, 142-156 (2009)

3. Prigione, A., Fauler, B., Lurz, R., Lehrach, H. \& Adjaye, J. The senescence-related mitochondrial/oxidative stress pathway is repressed in human induced pluripotent stem cells. Stem Cells 28, 721-733 (2010).

4. Rehman, J. Empowering self-renewal and differentiation: the role of mitochondria in stem cells. J. Mol. Med. (Berl.) 88, 981-986 (2010).

5. Prieto, J. et al. Dysfunctional mitochondrial fission impairs cell reprogramming Cell Cycle 15, 3240-3250 (2016).

6. Xiang, G. et al. BNIP3L-dependent mitophagy accounts for mitochondrial clearance during 3 factors-induced somatic cell reprogramming. Autophagy 13, 1543-1555 (2017).

7. Hom, J. R. et al. The permeability transition pore controls cardiac mitochondrial maturation and myocyte differentiation. Dev. Cell 21, 469-478 (2011).

8. Dai, D. F., Danoviz, M. E., Wiczer, B., Laflamme, M. A. \& Tian, R. Mitochondrial maturation in human pluripotent stem cell derived cardiomyocytes. Stem Cells Int. 2017, 5153625 (2017).

9. McQuibban, G. A., Lee, J. R., Zheng, L., Juusola, M. \& Freeman, M. Normal mitochondrial dynamics requires rhomboid-7 and affects Drosophila lifespan and neuronal function. Curr. Biol. 16, 982-989 (2006).

10. Deng, H., Dodson, M. W., Huang, H. \& Guo, M. The Parkinson's disease genes pink1 and parkin promote mitochondrial fission and/or inhibit fusion in Drosophila. Proc. Natl Acad. Sci. USA 105, 14503-14508 (2008).

11. Poole, A. C. et al. The PINK1/Parkin pathway regulates mitochondrial morphology. Proc. Natl Acad. Sci. USA 105, 1638-1643 (2008).
12. Yang, $Y$. et al. Pink1 regulates mitochondrial dynamics through interaction with the fission/fusion machinery. Proc. Natl Acad. Sci. USA 105, 7070-7075 (2008).

13. Wang, L. et al. Fatty acid synthesis is critical for stem cell pluripotency via promoting mitochondrial fission. EMBO J. 36, 1330-1347 (2017).

14. Suen, D. F., Norris, K. L. \& Youle, R. J. Mitochondrial dynamics and apoptosis. Genes Dev. 22, 1577-1590 (2008).

15. Chen, H. \& Chan, D. C. Mitochondrial dynamics in mammals. Curr. Top. Dev. Biol. 59, 119-144 (2004)

16. Chen, $\mathrm{H}$. et al. Mitochondrial fusion is required for mtDNA stability in skeletal muscle and tolerance of mtDNA mutations. Cell 141, 280-289 (2010).

17. Micchelli, C. A. \& Perrimon, N. Evidence that stem cells reside in the adult Drosophila midgut epithelium. Nature 439, 475-479 (2006).

18. Ohlstein, B. \& Spradling, A. The adult Drosophila posterior midgut is maintained by pluripotent stem cells. Nature 439, 470-474 (2006).

19. Fox, D. T. \& Spradling, A. C. The Drosophila hindgut lacks constitutively active adult stem cells but proliferates in response to tissue damage. Cell Stem Cell $\mathbf{5}$ 290-297 (2009).

20. Takashima, S., Mkrtchyan, M., Younossi-Hartenstein, A., Merriam, J. R. \& Hartenstein, $\mathrm{V}$. The behaviour of Drosophila adult hindgut stem cells is controlled by Wnt and Hh signalling. Nature 454, 651-655 (2008).

21. Takashima, S., Paul, M., Aghajanian, P., Younossi-Hartenstein, A. \& Hartenstein, V. Migration of Drosophila intestinal stem cells across organ boundaries. Development 140, 1903-1911 (2013).

22. Johansen, K. A., Green, R. B., Iwaki, D. D., Hernandez, J. B. \& Lengyel, J. A. The Drm-Bowl-Lin relief-of-repression hierarchy controls fore- and hindgut patterning and morphogenesis. Mech. Dev. 120, 1139-1151 (2003).

23. Takashima, S., Yoshimori, H., Yamasaki, N., Matsuno, K. \& Murakami, R. Cell-fate choice and boundary formation by combined action of Notch and engrailed in the Drosophila hindgut. Dev. Genes Evol. 212, 534-541 (2002).

24. Bach, E. A. et al. GFP reporters detect the activation of the Drosophila JAK STAT pathway in vivo. Gene. Expr. Patterns 7, 323-331 (2007).

25. Verstreken, $\mathrm{P}$. et al. Synaptic mitochondria are critical for mobilization of reserve pool vesicles at Drosophila neuromuscular junctions. Neuron 47, 365-378 (2005).

26. Cenveny, K. L., Tamura, Y., Zhang, Z., Jensen, R. E. \& Sesaki, H. Regulation of mitochondrial fusion and division. Trends Cell Biol. 17, 563-569 (2007).

27. Aghajanian, P., Takashima, S., Paul, M., Younossi-Hartenstein, A. \& Hartenstein, V. Metamorphosis of the Drosophila visceral musculature and its role in intestinal morphogenesis and stem cell formation. Dev. Biol. 420, 43-59 (2016).

28. Hay, B. A. \& Guo, M. Caspase-dependent cell death in Drosophila. Annu. Rev. Cell Dev. Biol. 22, 623-650 (2006)

29. Scaduto, R. C. Jr \& Grotyohann, L. W. Measurement of mitochondrial membrane potential using fluorescent rhodamine derivatives. Biophys. J. 76, 469-477 (1999).

30. Dimroth, P., Kaim, G. \& Matthey, U. Crucial role of the membrane potential for ATP synthesis by F(1)F(0) ATP synthases. J. Exp. Biol. 203, 51-59 (2000).

31. Paul, M. K. et al. Dynamic changes in intracellular ROS levels regulate airway basal stem cell homeostasis through Nrf2-dependent Notch signaling. Cell Stem Cell 15, 199-214 (2014).

32. Hochmuth, C. E., Biteau, B., Bohmann, D. \& Jasper, H. Redox regulation by Keap1 and Nrf2 controls intestinal stem cell proliferation in Drosophila. Cell Stem Cell 8, 188-199 (2011).

33. Biteau, B., Hochmuth, C. E. \& Jasper, H. JNK activity in somatic stem cells causes loss of tissue homeostasis in the aging Drosophila gut. Cell Stem Cell 3, 442-455 (2008).

34. Voog, J. et al. Escargot restricts niche cell to stem cell conversion in the Drosophila testis. Cell Rep. 7, 722-734 (2014).

35. Mathur, D., Bost, A., Driver, I. \& Ohlstein, B. A transient niche regulates the specification of Drosophila intestinal stem cells. Science 327, 210-213 (2010).

36. Takashima, S., Younossi-Hartenstein, A., Ortiz, P. A. \& Hartenstein, V. A nove tissue in an established model system: the Drosophila pupal midgut. Dev. Genes Evol. 221, 69-81 (2011).

37. Jiang, $\mathrm{H}$. et al. Cytokine/Jak/Stat signaling mediates regeneration and homeostasis in the Drosophila midgut. Cell 137, 1343-1355 (2009).

38. Affolter, M., Walldorf, U., Kloter, U., Schier, A. F. \& Gehring, W. J. Regional repression of a Drosophila POU box gene in the endoderm involves inductive interactions between germ layers. Development 117, 1199-1210 (1993).

39. Jiang, H., Grenley, M. O., Bravo, M. J., Blumhagen, R. Z. \& Edgar, B. A. EGFR/Ras/ MAPK signaling mediates adult midgut epithelial homeostasis and regeneration in Drosophila. Cell Stem Cell 8, 84-95 (2011). 
40. Hernandez-Garcia, D., Wood, C. D., Castro-Obregon, S. \& Covarrubias, L. Reactive oxygen species: a radical role in development? Free Radic. Biol. Med. 49, 130-143 (2010).

41. Szabadkai, G. et al. Mitochondrial dynamics and Ca2+signaling. Biochim. Biophys. Acta 1763, 442-449 (2006).

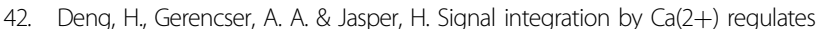
intestinal stem-cell activity. Nature 528, 212-217 (2015).
43. Chen, C. H. et al. A synthetic maternal-effect selfish genetic element drives population replacement in Drosophila. Science 316, 597-600 (2007).

44. Takashima, S. et al. Development of the Drosophila entero-endocrine lineage and its specification by the Notch signaling pathway. Dev. Biol. 353, 161-172 (2011). 